\title{
A management algorithm for adult patients with both brain oxygen and intracranial pressure monitoring: the Seattle International Severe Traumatic Brain Injury Consensus Conference (SIBICC)
}

Randall Chesnut ${ }^{1,2}$, , Sergio Aguilera ${ }^{3,4}$, Andras Buki ${ }^{5,6}$, Eileen Bulger ${ }^{7}$, Giuseppe Citerio ${ }^{8,9}$, D. Jamie Cooper ${ }^{10,11}$, Ramon Diaz Arrastia ${ }^{12}$, Michael Diringer ${ }^{13}$, Anthony Figaji ${ }^{14}$, Guoyi Gao ${ }^{15}$, Romer Geocadin ${ }^{16}$, Jamshid Ghajar ${ }^{17}$, Odette Harris ${ }^{18}$, Alan Hoffer ${ }^{19}$, Peter Hutchinson ${ }^{20}$, Mathew Joseph ${ }^{21}$, Ryan Kitagawa ${ }^{22}$, Geoffrey Manley ${ }^{23}$, Stephan Mayer ${ }^{24}$, David K. Menon ${ }^{25}$, Geert Meyfroidt ${ }^{26}$, Daniel B. Michael ${ }^{27}$, Mauro Oddo ${ }^{28}$, David Okonkwo ${ }^{29}$, Mayur Patel ${ }^{30}$, Claudia Robertson ${ }^{31}$, Jeffrey V. Rosenfeld ${ }^{32,33}$, Andres M. Rubiano ${ }^{34,35}$, Juan Sahuquillo ${ }^{36}$, Franco Servadei ${ }^{37,38}$, Lori Shutter ${ }^{39}$, Deborah Stein ${ }^{40}$, Nino Stocchetti ${ }^{41,42}$, Fabio Silvio Taccone ${ }^{43}$, Shelly Timmons ${ }^{44}$, Eve Tsai ${ }^{45}$, Jamie S. Ullman ${ }^{46}$, Paul Vespa ${ }^{47}$, Walter Videtta ${ }^{48}$, David W. Wright ${ }^{49}$, Christopher Zammit ${ }^{50}$ and Gregory W. J. Hawryluk ${ }^{51^{*}}$ (D)

(c) 2020 The Author(s)

\begin{abstract}
Background: Current guidelines for the treatment of adult severe traumatic brain injury (sTBI) consist of high-quality evidence reports, but they are no longer accompanied by management protocols, as these require expert opinion to bridge the gap between published evidence and patient care. We aimed to establish a modern sTBI protocol for adult patients with both intracranial pressure (ICP) and brain oxygen monitors in place.

Methods: Our consensus working group consisted of 42 experienced and actively practicing sTBI opinion leaders from six continents. Having previously established a protocol for the treatment of patients with ICP monitoring alone, we addressed patients who have a brain oxygen monitor in addition to an ICP monitor. The management protocols were developed through a Delphi-method-based consensus approach and were finalized at an in-person meeting.

Results: We established three distinct treatment protocols, each with three tiers whereby higher tiers involve therapies with higher risk. One protocol addresses the management of ICP elevation when brain oxygenation is normal. A second addresses management of brain hypoxia with normal ICP. The third protocol addresses the situation when both intracranial hypertension and brain hypoxia are present. The panel considered issues pertaining to blood transfusion and ventilator management when designing the different algorithms.
\end{abstract}

\footnotetext{
*Correspondence: ghawryluk@hsc.mb.ca

51 Section of Neurosurgery, University of Manitoba, GB1, 820 Sherbrook Street, Winnipeg, MB R3A 1R9, Canada

Full author information is available at the end of the article
}

\section{鱼 Springer}


Conclusions: These protocols are intended to assist clinicians in the management of patients with both ICP and brain oxygen monitors but they do not reflect either a standard-of-care or a substitute for thoughtful individualized management. These protocols should be used in conjunction with recommendations for basic care, management of critical neuroworsening and weaning treatment recently published in conjunction with the Seattle International Brain Injury Consensus Conference.

Keywords: Brain injury, Head trauma, Algorithm, Protocol, Consensus, Intracranial pressure, Brain oxygen, Tiers, Seattle, $\mathrm{SIBICC}, \mathrm{P}_{\mathrm{bt}} \mathrm{O}_{2}$

\section{Introduction}

The Seattle International Brain Injury Consensus Conference (SIBICC) [1] used a Delphi-method based consensus approach in an attempt to bridge the gap between the severe traumatic brain injury (sTBI) guidelines available for individual treatments [2] and the lack of evidence on how such treatments should be integrated into a practical management algorithm. The result, a protocol based on the formalized consensus of 42 international, multidisciplinary neurotrauma experts, provides class III evidence to guide the management of sTBI patients with intracranial pressure (ICP) as their only monitored brain-specific parameter [1]. During this process, the group expressed a desire to additionally address multi-modality monitoring if possible. They strongly supported brain tissue oxygen $\left(\mathrm{P}_{\mathrm{bt}} \mathrm{O}_{2}\right)$ monitoring as their first choice for the second parameter. Indeed, the evidence suggesting that sTBI clinical care informed by $\mathrm{P}_{b t} \mathrm{O}_{2}$ measures may lead to improved clinical outcomes is growing [3]. This paper thus presents the second product of the SIBICC meeting, an algorithm for managing adult sTBI patients based on combined monitoring of ICP and $\mathrm{P}_{\mathrm{bt}} \mathrm{O}_{2}$.

\section{Methods}

The methods were the same as used in the ICP-only work (see also supplementary methods) [1]. Fortytwo international intensivists, neurosurgeons, trauma surgeons, and emergency medicine physicians from six continents comprised the SIBICC consensus working group (CWG). We based panel selection on (a) $>10$ years clinical experience in sTBI; (b) current, active involvement in acute care management of sTBI patients; (c) representation of involved disciplines; (d) geographic diversity; (e) ability to commit time to the algorithm development process. We calculated panel size based on logistic considerations. Panelists completed conflict of interest forms relevant to STBI management. There were no conflicts mandating recusal of any participant.

The expressed focus of the effort was to design a management algorithm that would be acceptable to the panel and amenable to application in both neurological and general ICUs by physicians not specialized in neurointensive care. A priori, we specified that the threshold for consensus would be $80 \%$ or greater agreement by at least $80 \%$ of the voting panelists. Prior to the meeting, the CWG completed eight web-based surveys (SurveyMonkey Inc., San Mateo, CA, USA, www.surve ymonkey.com) to determine the algorithm design and focus, explore definitions and thresholds, operationally define treatment modalities, evaluate acceptability and tier assignments of treatment modalities, etc. We combined voting results with panelists' comments to iterate these surveys to maximize consensus and define areas requiring focus at the in-person meeting. It was during this process that the CWG elected to attempt development of an algorithm for combined ICP/ $/ \mathrm{P}_{b t} \mathrm{O}_{2}$ monitoring if time allowed.

The in-person SIBICC meeting occurred from the 5th to 7th of April 2019 in Seattle, Washington, USA. We used anonymous electronic voting and vote analysis (Electronic Media Services Inc., Gig Harbor Washington, USA, www.electronicmeetingservices.com). Professional, independent non-physician moderators facilitated group discussions. Unless specifically modified by the CWG, we limited the voting cycle to three iterations, interspersed with discussions. An element formed part of the final recommendations only if it attained $80 \%$ approval. Unresolved issues are reported as such. We used small group sessions to address complex issues, with the whole CWG modifying and voting on small group recommendations. Small group discussions relevant to these 'combined' algorithms focused particularly on the complexities of ventilator management and blood transfusion. All recommendations were incorporated verbatim into the final product.

The CWG recognized the notable time and effort that the Clinical Standardization Committee involved in designing the brain oxygen optimization in severe TBI-phase 3 (BOOST-3) trial had devoted to developing a study protocol to manage combined ICP and $\mathrm{P}_{\mathrm{bt}} \mathrm{O}_{2}$ abnormalities in the experimental limb of that randomized trial. Rather than ignoring the value of what they had produced in parallel, relevant aspects of the BOOST-3 protocol were reviewed during the 
development of the SIBICC algorithm. Discussion was facilitated as several CWG members overlapped both efforts. Notably, the BOOST-3 protocol was available for information only, and involved neither effort nor implication to adopt or merge any steps. The CWG acknowledges the cooperation of the whole BOOST-3 Clinical Standardization Committee in this process (see "Acknowledgements").

Donations solicited from industry and other interested parties funded the in-person meeting. In return, they were allowed to silently observe the conference, without any interaction with the panelists or the process. No donors or other outside parties influenced any portion of these recommendations.

\section{Results}

Consensus efforts generated a list of interventions viewed as fundamental to the care of sTBI patients and which should ideally be in place early in the course of care (Fig. 1). These 'tier zero' interventions are not dependent on the presence of ICP elevation. The CWG also generated a list of treatments that should not be used in the
Table 1 Treatment NOT recommended for use in the management of severe traumatic brain injury (when both ICP and $\mathrm{P}_{\mathrm{bt}} \mathrm{O}_{2}$ are monitored)

Mannitol by non-bolus continuous intravenous infusion

Scheduled infusion of hyperosmolar therapy (e.g., every $4-6$ h)

Lumbar CSF drainage

Furosemide

Routine use of steroids

Routine use of therapeutic hypothermia to temperatures below $35^{\circ} \mathrm{C}$ due to systemic complications

High-dose propofol to attempt burst suppression

Decreasing $\mathrm{P}_{\mathrm{a}} \mathrm{CO}_{2}$ below $30 \mathrm{mmHg} / 4.0 \mathrm{kPa}$

Routinely raising CPP above $90 \mathrm{mmHg}$

Barbiturates as treatment for low $\mathrm{P}_{\mathrm{bt}} \mathrm{O}_{2}$ unless barbiturates are otherwise indicated

Hypothermia as treatment for low $\mathrm{P}_{\mathrm{bt}} \mathrm{O}_{2}$ unless hypothermia is otherwise indicated

Hypercarbia in "type D" patients

$C P P$ cerebral perfusion pressure, ICP intracranial pressure, $k$ Pa kiloPascals, $\mathrm{P}_{a} \mathrm{CO}_{2}$ arterial partial pressure of carbon dioxide, $\mathrm{P}_{b t} \mathrm{O}_{2}$ brain tissue partial pressure of oxygen

\section{Expected Interventions:}

- Admission to ICU

- Endotracheal intubation and mechanical ventilation

- Serial evaluations of neurological status and pupillary reactivity

- Elevate HOB $30-45^{\circ}$

- Analgesia to manage signs of pain (not ICP or $\mathrm{P}_{\mathrm{bt}} \mathrm{O}_{2}$ directed)

- Sedation to prevent agitation, ventilator asynchrony, etc. (not ICP or $\mathrm{P}_{\mathrm{bt}} \mathrm{O}_{2}$ directed)

- Temperature management to prevent fever Measure core temperature

Treat core temperature above $38^{\circ} \mathrm{C}$

\section{Recommended Interventions:}

- Insertion of a central line

- End-tidal $\mathrm{CO}_{2}$ monitoring
- Consider anti-seizure medications for $1 \mathrm{w}$ only (in the absence of an indication to continue)

- Maintain CPP initially $\geq 60 \mathrm{mmHg}$

- Maintain $\mathrm{Hb}>7 \mathrm{~g} / \mathrm{dL}$

- Avoid hyponatremia

- Optimize venous return from head

(eg. keep head midline, ensure cervical collars are no too tight)

- Arterial line continuous blood pressure monitoring

- Maintain $\mathrm{S}_{\mathrm{p}} \mathrm{O}_{2} \geq 94 \%$

Fig. 1 Consensus-based basic severe traumatic brain injury care for patients with an ICP and brain oxygen monitor in situ. These are basic treatments recommended as fundamental to the care of patients with sTBI, to be initiated ("Expected interventions") or considered ("Recommended interventions") upon ICU admission of a patient with both an ICP and brain oxygen monitor, regardless of the measured values. $\mathrm{CO}_{2}$ Carbon dioxide, CPP cerebral perfusion pressure, $\mathrm{Hg}$ hemoglobin, $\mathrm{HOB}$ head of bed, ICP intracranial pressure, ICU intensive care unit, $\mathrm{spO}_{2}$ arterial oxygen saturation 


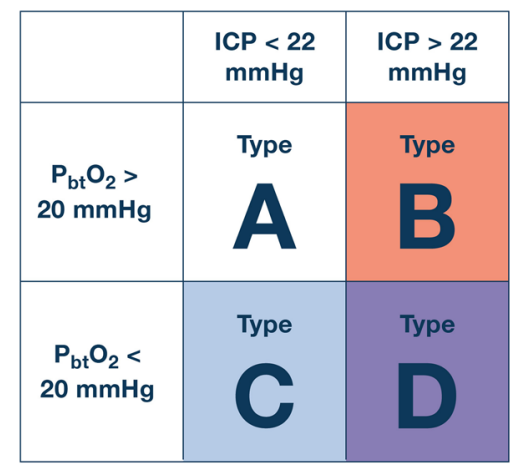

Fig. 2 This matrix provides the schema for the 4 clinical conditions encountered in patients with both ICP and brain oxygen monitors in situ. Type A reflects normal values for both monitors and does not require treatment. Type $B$ involves ICP elevation but normal brain oxygen values; we propose a distinct treatment algorithm for such patients than in those with ICP elevation and unknown $\mathrm{P}_{b t} \mathrm{O}_{2}$ values. Type $C$ patients have hypoxic brains but normal ICP and Type D patients have both brain hypoxia and ICP elevation. An ICP of 22 $\mathrm{mmHg}$ discriminates normal (lower) and abnormal (higher) values while $\mathrm{P}_{\mathrm{bt}} \mathrm{O}_{2}$ values of $20 \mathrm{mmHg}$ discriminates normal (higher) and abnormal (lower) values. ICP intracranial pressure, $\mathrm{P}_{\mathrm{bt}} \mathrm{O}_{2}$ partial pressure of brain oxygen

care of patients with sTBI (Table 1), except in special circumstances. The additional algorithms provided here use the same three-tier algorithm structure as in the ICPonly algorithm development [1]. To accommodate the combination of two monitors, a schema consisting of a $2 \times 2$ table combining the permutations of ICP and $\mathrm{P}_{b t} \mathrm{O}_{2}$ status was used (Fig. 2). The CWG developed individual management algorithms for the three types with abnormal monitored values: Fig. 3 presents the algorithm for Type $\mathrm{B}$ (abnormal ICP and normal $\mathrm{P}_{\mathrm{bt}} \mathrm{O}_{2}$ ), Fig. 4 presents the algorithm for Type $\mathrm{C}$ (normal ICP and abnormal $\mathrm{P}_{\mathrm{bt}} \mathrm{O}_{2}$ ), and Fig. 5 presents the algorithm for Type $\mathrm{D}$ (both ICP and $\mathrm{P}_{\mathrm{bt}} \mathrm{O}_{2}$ abnormal).

The CWG also provides inter-tier recommendations (Figs. 3,4,5) and guidance on Critical Neuroworsening (Fig. 6) to assist in evaluating and managing patients requiring increased therapeutic intensity.

\section{Discussion}

As with the first SIBICC effort that produced a management algorithm for adult sTBI patients with ICP monitoring alone [1], this work uses Delphi process-based mechanics to provide basic evidence guiding integration of individual treatment modalities into management algorithms for patients with combined ICP/ $/ \mathrm{P}_{\mathrm{bt}} \mathrm{O}_{2}$ monitoring. The process amalgamated the practice-based recommendations of 42 international, experienced, clinically active neurotrauma practitioners from those disciplines involved in acute post-traumatic (ICU) care by means of consensus achieved with blinded voting. Such formalized integration of expert opinion provides the most basic level of evidence towards organizing and standardizing care, relevant to all neurotrauma practitioners but particularly to centers not specifically expert in the management of sTBI or those considering initiating combined $\mathrm{ICP} / \mathrm{P}_{\mathrm{bt}} \mathrm{O}_{2}$ monitoring.

Given the class III status of this evidence, these algorithms should be considered as a suggested treatment method without proven superiority over other applicable methods. They represent a safe and modern approach to sTBI care. They are not a standard of care nor are they likely to represent the best treatment approach in a given instance. They are not legally binding and they are not designed as quality assurance monitoring tools. They do not represent the approach of any individual CWG member and should not be substituted for thoughtful clinical judgment. Variability within individual patients or patient cohorts (e.g. center variations) may necessitate local adaptation, which is entirely within the nature of this offering.

\section{Algorithm structure}

The combination of ICP and $\mathrm{P}_{\mathrm{bt}} \mathrm{O}_{2}$ monitoring lends itself to several possible protocol structures. One option is to conceptualize ICP and $\mathrm{P}_{\mathrm{bt}} \mathrm{O}_{2}$ management separately and to present them as distinct pathways, as has been done for the paediatric sTBI guidelines $[4,5]$. The other is to maintain integration of the two monitors and create separate algorithms for the three pathologic combinations of ICP and $\mathrm{P}_{\mathrm{bt}} \mathrm{O}_{2}$ status. Our panel felt that when high ICP and low $\mathrm{P}_{b t} \mathrm{O}_{2}$ are present concurrently ideal management would not simply reflect a simple combination of care provided when high ICP and low $\mathrm{P}_{\mathrm{bt}} \mathrm{O}_{2}$ each exist in isolation. In particular, the CWG felt that mechanical ventilation requires distinct management when both high ICP and low $\mathrm{P}_{b t} \mathrm{O}_{2}$ are present concurrently. In the interest of supporting precision medicine and to ease clinical application by providing specific, separate protocols for individual pathological combinations, we chose to present three distinct algorithms (Figs. 2,3,4,5). For a given combination (type $\mathrm{B}, \mathrm{C}$, or $\mathrm{D}$ ), the relevant protocol should be applied. Changes in clinical status should prompt adjustment to the newly germane algorithm as well as a thoughtful clinical approach.

\section{Conditions of tiered treatment}

The use of tiers attempts to balance the benefits and efficacy of an agent against risks inherent to its use. General clinical management is considered tier zero. Treatment 


\section{Type B}

\section{ICP Elevated - Brain Oxygenation Normal}

Tier 1

- Maintain CPP 60-70 mm Hg

- Increase analgesia to lower ICP

- Increase sedation to lower ICP

- Maintain $\mathrm{PaCO}_{2}$ at low end of normal (35-38 mm Hg/4.7-5.1 kPa)

- Mannitol by intermittent bolus $(0.25-1.0 \mathrm{~g} / \mathrm{kg})$
- Hypertonic saline by intermittent bolus*

- CSF drainage if EVD in situ

- Consider placement of EVD to drain CSF

if parenchymal probe used initially

- Consider anti-seizure prophylaxis for 1 week only (unless indication to continue)

- Consider EEG monitoring

\section{Tier 2}

- Mild hypocapnia (range 32-35 mmHg/4.3-4.7 kPa)

- Neuromuscular paralysis in adequately sedated patients if efficacious in lowering ICP**

- Perform MAP challenge to assess cerebral autoregulation and guide MAP and CPP goals in individual patients†

- Should be performed under direct supervision of a physician who can assess response and ensure safety

- No other therapeutic adjustments (ie. sedation) should be performed during the MAP challenge

- Initiate or titrate a vasopressor or inotrope to increase MAP by $10 \mathrm{mmHg}$ for not more than 20 minutes

- Monitor and record key parameters (MAP, CPP, ICP and $\mathrm{P}_{b t} \mathrm{O}_{2}$ ) before during and after the challenge

- Adjust vasopressorlinotrope dose based on study findings

- Raise CPP with fluid boluses, vasopressors and/or inotropes to lower ICP when autoregulation is intact

\section{Tier 3}

- Pentobarbital or Thiopentone coma titrated to ICP control if efficacious $\ddagger$

- Secondary decompressive craniectomy
- Mild hypothermia $\left(35-36^{\circ} \mathrm{C}\right)$ using active cooling measures

- Hyperventilation to $\mathrm{P}_{\mathrm{a}} \mathrm{CO}_{2}$ of $30-32 \mathrm{mmHg} / 4.0-4.3 \mathrm{kPa}$
- Re-examine the patient and consider repeat CT to re-evaluate intracranial pathology

- Reconsider surgical options for potentially surgical lesions

- Consider extracranial causes of ICP elevation

- Review that basic physiologic parameters are in desired range (e.g. CPP, blood gas values)

- Consider consultation with higher level of care if applicable for your health care system

\footnotetext{
* We recommend using sodium and osmolality limits of $155 \mathrm{mEq} / \mathrm{L}$ and of $320 \mathrm{mEq} / \mathrm{L}$ respectively as administration limits for both mannitol and hypertonic saline.

${ }^{\star}$ We recommend a trial dose of neuromuscular paralysis and only proceeding to a continuous infusion when efficacy is demonstrated.

† Rosenthal G. et al 2011

$\ddagger$ Barbiturate administration should only be continued when a beneficial effect on ICP is demonstrated.

Titrate barbiturate to achieve ICP control but do not exceed the dose which achieves burst suppression.

Hypotension must be avoided when barbiturates are administered.
}

Fig. 3 Consensus-based algorithm for the management of severe traumatic brain injury with intracranial hypertension and normal brain oxygenation. Lower tier treatments are viewed as having a more favorable side effect profile than higher tiers and generally should be employed first. Intertier recommendations encourage patient reassessment for remediable causes of treatment resistance. See text for details. $C P P$ cerebral perfusion pressure, EEG electroencephalogram, EVD external ventricular drain, ICP intracranial pressure, $K P a$ kiloPascals, $M A P$ mean arterial pressure, $P_{a} C O_{2}$ arterial partial pressure of carbon dioxide

of intracranial hypertension or brain hypoxia will generally begin at tier one. Movement to higher tiers reflects increasingly aggressive interventions. Here, treatments in any given tier are considered equivalent, with the selection of one treatment over another based on individual patient characteristics and physician discretion. During any given episode being addressed, multiple items from a single tier can be trialed individually or in combination with the goal of a rapid response. The provider should maintain awareness of the duration of any episode and consider moving to more aggressive interventions in a higher tier quickly if the patient is not responding. In 


\section{Type C}

\section{ICP Normal - Brain Hypoxic}

Tier 1

- Maintain CPP 60-70 mm Hg

- Increase CPP to a maximum of $70 \mathrm{mmHg}$ with fluid, vasopressors and/or inotropes

- Maintain $\mathrm{PaCO}_{2}>(35 \mathrm{mmHg} / 4.7 \mathrm{kPa})$

- If $\mathrm{P}_{\mathrm{a}} \mathrm{O}_{2}$ is already in desired ranged, further increase $\mathrm{PaO}_{2}$ by increasing $\mathrm{F}_{\mathrm{i}} \mathrm{O}_{2}$ to $60 \%$

- Consider EEG monitoring

Tier 2

- Ventilator management to increase $\mathrm{P}_{\mathrm{a}} \mathrm{O}_{2}$ as high as $150 \mathrm{mmHg} / 20 \mathrm{kPa}$

- Decrease ICP to a threshold $<22 \mathrm{mmHg}$

- Consider CSF drainage

- Increase sedation to improve mechanical ventilation and $\mathrm{Pbt}_{\mathrm{bt}} \mathrm{O}_{2}$

- Neuromuscular paralysis in adequately sedated patients if efficacious in increasing $\mathrm{Pbt}_{\mathrm{bt}} \mathrm{O}_{2}$ *

- Perform MAP challenge to assess cerebral autoregulation and guide MAP and CPP goals in individual patients $†$

- Should be performed under direct supervision of a physician who can assess response and ensure safety

- No other therapeutic adjustments (ie. sedation) should be performed during the MAP challenge

- Initiate or titrate a vasopressor or inotrope to increase MAP by $10 \mathrm{mmHg}$ for not more than 20 minutes

- Monitor and record key parameters (MAP, CPP, ICP and $\mathrm{P}_{b t} \mathrm{O}_{2}$ ) before during and after the challenge

- Adjust vasopressorlinotrope dose based on study findings

- Raise CPP to increase PbtO2 when supported by MAP Challenge

- Increase CPP above $70 \mathrm{mmHg}$ with fluid boluses, vasopressors and/or inotropes **

- Re-examine the patient and consider repeat $\mathrm{CT}$ to re-evaluate intracranial pathology

- Reconsider surgical options for potentially surgical lesions

- Consider extracranial causes of ICP elevation

- Review that basic physiologic parameters are in desired range (e.g. CPP, blood gas values)

- Consider consultation with higher level of care if applicable for your health care system

\section{Tier 3}

- Increase $\mathrm{P}_{\mathrm{a}} \mathrm{CO}_{2}$ to $45-50 \mathrm{mmHg} / 6.0-6.7 \mathrm{kPa}$ (but avoid intracranial hypertension)

- Consider normobaric hyperoxia to a $\mathrm{P}_{\mathrm{a}} \mathrm{O}_{2}$ above $150 \mathrm{mmHg} / 20 \mathrm{kPa}$

- If $\mathrm{P}_{\mathrm{bt}} \mathrm{O}_{2}$ remains $<20 \mathrm{mmHg}$ despite $\mathrm{P}_{\mathrm{a}} \mathrm{O}_{2}$ and CPP/MAP optimization, consider transfusing 1 unit of PRBCs if $\mathrm{Hgb}<9 \mathrm{~g} / \mathrm{L}$

* We recommend a trial dose of neuromuscular paralysis and only proceeding to a continuous infusion when efficacy is demonstrated.

${ }^{\star *}$ Careful monitoring for respiratory complications is required when CPP is raised above $70 \mathrm{mmHg}$ (Robertson et al, 1999)

† Rosenthal G. et al 2011

Fig. 4 Consensus-based algorithm for the management of severe traumatic brain injury with brain hypoxia and normal intracranial pressure. Lower tier treatments are viewed as having a more favorable side effect profile than higher tiers and generally should be employed first. Inter-tier recommendations encourage patient reassessment for remediable causes of treatment resistance. See text for details. CPP cerebral perfusion pressure, EEG electroencephalogram, EVD external ventricular drain, ICP intracranial pressure, $\mathrm{KPa}$ kiloPascals, MAP mean arterial pressure, $P_{\mathrm{a}} \mathrm{CO}_{2}$ arterial partial pressure of carbon dioxide

some cases, it might be preferable to skip one or more tiers (e.g. choosing to decompress a patient with midline shift due to hemispheric swelling and very high initial ICP). No individual agent or combination thereof is critical to success in managing TBI. Clinical judgment must always determine the final management strategy.

Tier-zero (Fig. 1) recommendations apply to sTBI patients who are admitted to an ICU in whom the 


\section{Type D}

\section{ICP Elevated - Brain Hypoxic}

Tier 1

- Maintain CPP 60-70 mm Hg

- Increase CPP to a maximum of $70 \mathrm{mmHg}$ with fluid, vasopressors and/or inotropes

- Increase analgesia to lower ICP/improve ventilation and $\mathrm{P}_{b t} \mathrm{O}_{2}$

- Increase sedation to lower ICP/improve ventilation and $\mathrm{P}_{\mathrm{bt}} \mathrm{O}_{2}$

- Maintain $\mathrm{PaCO}_{2}>35 \mathrm{mmHg} / 4.7 \mathrm{kPa}$

- Mannitol by intermittent bolus $(0.25-1.0 \mathrm{~g} / \mathrm{kg})$

- Hypertonic saline by intermittent bolus*
- CSF drainage if EVD in situ

- Consider placement of EVD to drain CSF if parenchymal probe used initially

- If $\mathrm{P}_{2} \mathrm{O}_{2}$ is already in desired range, further increase $\mathrm{P}_{\mathrm{a}} \mathrm{O}_{2}$ by increasing $\mathrm{F}_{\mathrm{i}} \mathrm{O}_{2}$ to $60 \%$

- Consider anti-seizure prophylaxis for 1 week only (unless indication to continue)

- Consider EEG monitoring

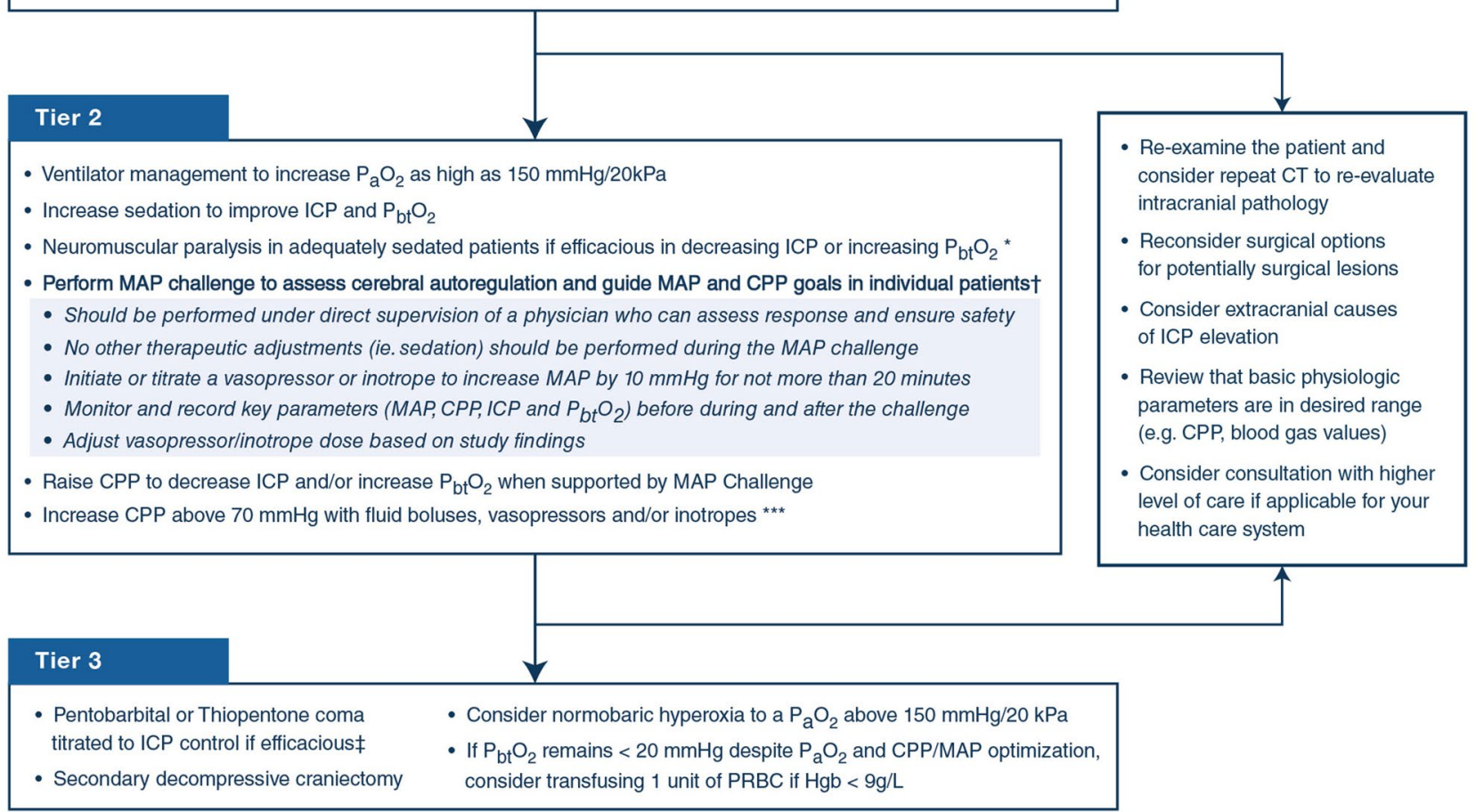

* We recommend using sodium and osmolality limits of $155 \mathrm{mEq} / \mathrm{L}$ and of $320 \mathrm{mEq} / \mathrm{L}$ respectively as administration limits for both mannitol and hypertonic saline.

** We recommend a trial dose of neuromuscular paralysis and only proceeding to a continuous infusion when efficacy is demonstrated.

† Rosenthal G. et al 2011

‡ Barbiturate administration should only be continued when a beneficial effect on ICP is demonstrated.

Titrate barbiturate to achieve ICP control but do not exceed the dose which achieves burst suppression.

Hypotension must be avoided when barbiturates are administered.

Fig. 5 Consensus-based algorithm for the management of severe traumatic brain injury with intracranial hypertension and brain hypoxia. Lower tier treatments are viewed as having a more favorable side effect profile than higher tiers and generally should be employed first. Inter-tier recommendations encourage patient reassessment for remediable causes of treatment resistance. See text for details. CPP cerebral perfusion pressure, EEG electroencephalogram, EVD external ventricular drain, ICP intracranial pressure, $K P a$ kiloPascals, $M A P$ mean arterial pressure, $P_{a} C O_{2}$ arterial partial pressure of carbon dioxide

decision to concurrently monitor ICP and $\mathrm{P}_{\mathrm{bt}} \mathrm{O}_{2}$ has been made. Management recommendations for sTBI patients without ICP monitoring are published elsewhere [6].
The goal of tier-zero is to establish a stable, neuroprotective physiologic baseline regardless of eventual ICP or $\mathrm{P}_{\mathrm{bt}} \mathrm{O}_{2}$ readings. Tier-zero sedatives and analgesics 


\section{Critical Neuroworsening}

A serious deterioration in clinical neurologic status such as:

- Spontaneous decrease in the GCS motor score of $\geq 1$ points (compared with the previous examination)

- New decrease in pupillary reactivity

- New pupillary asymmetry or bilateral mydriasis

- New focal motor deficit

- Herniation syndrome or Cushing's Triad which requires an immediate physician response

\section{Response to Critical Neuroworsening}

- Emergent evaluation to identify possible cause* of neuroworsening

- If herniation is suspected:

- empiric treatment

- hyperventilation ${ }^{* *}$

- bolus of hypertonic solution

- consider emergent imaging or other testing

- rapid escalation of treatment

* Possible causes of neuroworsening include:

$\begin{array}{lll}\text { - expanding intracranial } & \bullet \text { medical comorbidity } & \bullet \text { CNS infection } \\ \text { mass lesion } & \text { - medication effect } & \bullet \text { infection or sepsis } \\ \text { - cerebral edema } & \bullet \text { impaired renal or } & \bullet \text { substance withdrawal } \\ \text { - elevated ICP } & \text { hepatic function } & \bullet \text { dehydration } \\ \text { - stroke } & \text { - systemic hypotension } & \bullet \text { hyper or hypothermia } \\ \text { - electrolyte or other } & \text { - seizure or post-ictal state } & \\ \text { metabolic disturbance } & \text { • hypoxemia/tissue hypoxia } & \\ \text { ** the hyperventilation } \mathrm{PaCO}_{2} \mathrm{CO}_{2} \text { limit of } 30 \mathrm{mmHg} / 4.0 \mathrm{kPa} \text { does not apply here }\end{array}$

Fig. 6 Critical neuroworsening and its management. SIBICC definition (upper box), response (middle box) and a list of suggested differential diagnoses (bottom) surrounding critical neurological deterioration (critical neuroworsening). CNS central nervous system, GCS Glasgow Coma Scale, ICP intracranial pressure

target comfort and ventilator tolerance rather than ICP or $\mathrm{P}_{\mathrm{bt}} \mathrm{O}_{2}$. Temperature management targets the avoidance of fever (defined by the CWG as core temperature $\left.>38^{\circ} \mathrm{C}\right)$. Consistent with the BTF Guidelines [2], the minimal cerebral perfusion pressure (CPP) threshold is $60 \mathrm{~mm} \mathrm{Hg}$.

The algorithm for type B patients differs from that recommended for the management of intracranial hypertension in patients with ICP-monitoring alone [1] due to available $\mathrm{P}_{b t} \mathrm{O}_{2}$ evidence that cerebral hypoxia is not present. Although tiers one and two are the same as for the ICP-only algorithm, this difference is reflected in the recommendation that hyperventilation to a $\mathrm{P}_{\mathrm{a}} \mathrm{CO}_{2}$ of $30-32 \mathrm{mmHg} / 4.0-4.3 \mathrm{kPa}$ can be considered in tier three. The CWG does not recommend either hypertonic saline or mannitol as preferable and uses the same limits for serum sodium and osmolality for both agents. They recommend CSF drainage if an external ventricular drain is available and consideration of placing one if other means are used to monitor ICP. They also recommend considering the possibility of seizures as the etiology of intracranial hypertension in tier one.

The CWG recommends consideration of a trial of neuromuscular blockade (with continuation if it is effective) as a tier two intervention. They also support mild hyperventilation $\left(\mathrm{P}_{\mathrm{a}} \mathrm{CO}_{2} 32-35 \mathrm{~mm} \mathrm{Hg} / 4.7 \mathrm{kPa}\right)$ at this level. The CWG also recommends the consideration of autoregulation testing via CPP manipulation to 
determine whether CPP augmentation might be applicable. The MAP challenge is performed under stable conditions to prevent confounding (e.g., no other active changes in care should be made during the challenge, including adjustments in sedation, analgesia, EVD drainage or other physiological parameters). To perform the challenge [7]:

- Record baseline monitor parameters at the beginning of the challenge (e.g., ICP, MAP and CPP).

- Initiate or titrate a vasopressor to increase the MAP by $10 \mathrm{~mm} \mathrm{Hg}$ for up to $20 \mathrm{~min}$.

- Observe the interaction between the MAP, ICP, CPP and $\mathrm{P}_{\mathrm{bt}} \mathrm{O}_{2}$ during the challenge.

- Record monitor parameters at the end of the challenge.

- Evaluate the observed responses and recorded values for evidence of sPAR status. Disrupted sPAR will present as a sustained increase in ICP with MAP elevation.

- Adjust the target MAP back to baseline (disrupted sPAR) or to the chosen new, elevated target (intact SPAR).

As noted above, tier three treatment in type B parallels that for ICP-monitor-only sTBI patients, with the exception that moderate hyperventilation $\left(\mathrm{P}_{\mathrm{a}} \mathrm{CO}_{2}\right.$ $30-32 \mathrm{mmHg} / 4.0-4.3 \mathrm{kPa}$ ) is also included.

Type $\mathrm{C}$ treatments include interventions known to directly or indirectly improve $\mathrm{P}_{\mathrm{bt}} \mathrm{O}_{2}$ values, largely based upon the collective experience of the expert CWG. A great deal of discussion surrounded augmentation of oxygenation in terms of benefit versus toxicity and the possibility that higher monitor readings may not parallel improved oxygen availability when $\mathrm{F}_{\mathrm{i}} \mathrm{O}_{2}$ or $\mathrm{P}_{\mathrm{a}} \mathrm{O}_{2}$ are pushed very high. $\mathrm{F}_{\mathrm{i}} \mathrm{O}_{2}$ can effectively increase $\mathrm{P}_{\mathrm{bt}} \mathrm{O}_{2}$ values. Although the CWG approved upward $\mathrm{F}_{\mathrm{i}} \mathrm{O}_{2}$ adjustment to $60 \%$ at tier one, further oxygenation manipulation was left to the discretion of the physician in terms of manipulation of ventilator dynamics, PEEP, $\mathrm{F}_{\mathrm{i}} \mathrm{O}_{2}$, etc. Therefore, at tier two, elevating the $\mathrm{P}_{\mathrm{a}} \mathrm{O}_{2}$ up to $150 \mathrm{~mm} \mathrm{Hg}$ is recommended but the means is meant to be fine-tuned to the patient. Further elevation of the $\mathrm{P}_{\mathrm{a}} \mathrm{O}_{2}$ was controversial but the final decision was to recommend normobaric hyperoxia above $150 \mathrm{~mm} \mathrm{Hg}$ at tier three.

The notion of blood transfusion to ameliorate low $\mathrm{P}_{b t} \mathrm{O}_{2}$ values also generated significant discussion. Harm from the transfusion of blood products is increasingly recognized $[8,9]$ and many on the panel felt that transfusion had little impact on $\mathrm{P}_{\mathrm{bt}} \mathrm{O}_{2}$ values in their experience. The CWG finally settled on limiting blood transfusion to tier three, recommending consideration of transfusing one unit of PRBCs in the setting of a $\mathrm{P}_{b t} \mathrm{O}_{2}<20 \mathrm{~mm} \mathrm{Hg}$ and an $\mathrm{Hgb}<9 \mathrm{~g} / \mathrm{L}$. It was acknowledged that blood transfusion would be a stronger consideration in patients with active organ ischemia (eg. cardiac ischemia).

Other recommendations in type $\mathrm{C}$ differ from those in type $\mathrm{B}$ patients. First-tier recommendations include setting the target CPP at the upper limit of the generally accepted range of $60-70 \mathrm{~mm} \mathrm{Hg}$ as blood pressure augmentation can be an effective strategy for increasing $\mathrm{P}_{\mathrm{bt}} \mathrm{O}_{2}$. The avoidance of hypocarbia is also stressed in tier one; hypercarbia induces vasodilation which can improve $\mathrm{P}_{\mathrm{bt}} \mathrm{O}_{2}$ though this strategy increases intracranial blood volume and risks ICP elevation. At tier two, the CWG supported consideration of setting an ICP threshold below the general target of $22 \mathrm{~mm} \mathrm{Hg}$ (acknowledging that this group does not have intracranial hypertension by definition). They also allowed compliance manipulation via CSF drainage.

Type $\mathrm{D}$ recommendations combine ICP and $\mathrm{P}_{b t} \mathrm{O}_{2}$ treatments, but aim to limit those treatments that would exacerbate either pathology. At tier one, therefore, although the CWG supported elevation of the $\mathrm{F}_{\mathrm{i}} \mathrm{O}_{2}$ to $60 \%$ and setting the CPP threshold to the upper limit of the normal range (i.e., $70 \mathrm{~mm} \mathrm{Hg}$ ), they recommended against hyperventilation to any degree which could worsen $\mathrm{P}_{b t} \mathrm{O}_{2}$. In tier two, the CWG recommended raising the $\mathrm{P}_{\mathrm{a}} \mathrm{O}_{2}$ to as high as $150 \mathrm{~mm} \mathrm{Hg}$ in addition to considering neuromuscular blockade and CPP manipulation based on autoregulation testing but did not support lowering the ICP threshold. At tier three, they combined ICP-based recommendations for pentobarbital/ thiopentone coma or decompressive craniectomy with $\mathrm{P}_{\mathrm{bt}} \mathrm{O}_{2}$-based treatments including normobaric hyperoxia to above $150 \mathrm{~mm} \mathrm{Hg}$ and limited transfusion. Notably, decompressive craniectomy can enable more aggressive $\mathrm{P}_{\mathrm{bt}} \mathrm{O}_{2}$ augmentation strategies such as hypercarbia which can exacerbate ICP; it can also mitigate the intracranial hypertension associated with MAP augmentation in patients who are not autoregulating.

The CWG carried forward those items that they did not recommend for treating intracranial hypertension in ICP-Only patients (Table 1 ). They added three $\mathrm{P}_{\mathrm{bt}} \mathrm{O}_{2}$-specific items to this list. They recommended against using high-dose barbiturates or cooling specifically for the management of $\mathrm{P}_{b t} \mathrm{O}_{2}$ though these therapies can be thoughtfully administered for other indications. They also recommended against routinely using hypercarbia $\left(\mathrm{P}_{\mathrm{a}} \mathrm{CO}_{2}>45 \mathrm{~mm} \mathrm{Hg} / 6.0 \mathrm{kPa}\right.$ in type $\mathrm{D}$ patients given the risk of elevating ICP.

\section{Inter-tier recommendations}

Stepping to a higher tier is a potential indicator of increased disease severity. As higher tiers represent 
interventions with increased associated risks, the CWG recommends reassessing the patient's basic intra-and extra-cranial physiologic status and reconsidering the surgical status of intracranial mass lesions (e.g. contusions) not previously considered operative. If the patient is at a non-specialist center at the point of upward tier advancement, the CWG recommended considering consultation with and potential transfer to a TBI center with increased resources if possible within the regional healthcare environment. When desired, transfer is best completed before clinical decline precludes it.

Critical Neuroworsening represents a specific situation of critical deterioration requiring emergent evaluation and management. 'Neuroworsening' was first defined as a potential intermediate-outcome variable for TBI trials [10], it was adapted for the BEST:TRIP trial [11] and subsequent management studies for limited resource environments [6]. The CWG modified the criteria, terming the new criteria 'Critical Neuroworsening' and added it to promote its recognition as a critical event and guide expeditious evaluation and consideration of empiric therapy.

\section{Summary}

As with the recently published SIBICC ICP-Only algorithm [1], this effort provides a bridge between the academic value of formal evidence reports [2] and practical, bedside management. It relies on "medicine-based-evidence" gleaned from 42 experienced, currently practicing experts, rigorously synthesized using a Delphi-methodbased consensus process. The CWG agreed that $\mathrm{P}_{b t} \mathrm{O}_{2}$ should be the second monitored variable after ICP, representing a step toward multi-modality monitoring. These recommendations represent the lowest level of evidence - a form of multi-physician curbside consult-and are presented as a framework for adoption or adaption by trauma systems or medical centers toward developing organized, protocol-based approaches to adult sTBI management. They are not binding and should not be viewed as the only or necessarily the best method of management of sTBI. They are offered as guidance only, as a first attempt at filling a gap in the current clinical literature.

\section{Electronic supplementary materia}

The online version of this article (https://doi.org/10.1007/s00134-019-05900-x) contains supplementary material, which is available to authorized users.

\section{Abbreviations}

BTF: Brain Trauma Foundation; CNS: Central nervous system; CPP: Cerebral perfusion pressure; CSF: Cerebrospinal fluid; CT: Computed tomography; DI: Diffuse injury as defined in the Marshall CT Head Score; EEG: Electroencephalogram; EML: Evacuated mass lesion as defined in the Marshall CT Head Score; EVD: External ventricular drain; GCS: Glasgow Coma Scale; Hg: Hemoglobin; HOB: Head of bed; ICE: Imaging and clinical examination; ICP: Intracranial pressure; ICU: Intensive care unit; KPa: KiloPascals; MAP: Mean arterial pressure;
$\mathrm{P}_{\mathrm{a}} \mathrm{CO}_{2}$ : Arterial partial pressure of carbon dioxide; $\mathrm{P}_{\mathrm{bt}} \mathrm{O}_{2}$ : Partial pressure of brain tissue oxygen; $\mathrm{SpO}_{2}$ : Arterial oxygen saturation.

\section{Author details}

${ }^{1}$ Department of Neurological Surgery, Harborview Medical Center, University of Washington, 325 Ninth Ave, Mailstop 359766, Seattle, Washington 98104-2499, USA. ${ }^{2}$ Department of Orthopaedic Surgery, Harborview Medical Center, University of Washington, 325 Ninth Ave, Mailstop 359766, Seattle, Washington 98104-2499, USA. ${ }^{3}$ Almirante Nef Naval Hospital, Viña del Mar, Chile. ${ }^{4}$ Valparaiso University, Valparaiso, Chile. ${ }^{5}$ Department of Neurosurgery, Medical School and Szentágothai Research Centre, Ifúság útja 20, 7624 Pécs, Hungary. ${ }^{6}$ University of Pécs, Pécs, Hungary. ${ }^{7}$ Department of Surgery, Harborview Medical Center, University of Washington, 325 Ninth Ave, Seattle, WA 98104-2499, USA. ${ }^{8}$ School of Medicine and Surgery, University of MilanBicocca, Milan, Italy. ${ }^{9}$ Neuro-Intensive Care, Department of Emergency and Intensive Care, ASST, San Gerardo Hospital, Monza, Italy. ${ }^{10}$ Intensive Care Medicine, Australian and New Zealand Intensive Care Research Centre, Monash University, Monash, Australia. ${ }^{11}$ Department of Intensive Care, Alfred Hospital, Melbourne, VIC, Australia. ${ }^{12}$ University of Pennsylvania Perelman School of Medicine, Penn Presbyterian Medical Center, 51 North 39th Street, Philadelphia, PA 19104, USA. ${ }^{13}$ Department of Neurology, Barnes-Jewish Hospital, Washington University School of Medicine, 1 Barnes-Jewish Hospital Plaza, St. Louis, MO 63110, USA. ${ }^{14}$ Division of Neurosurgery and Neuroscience Institute, University of Cape Town, H53 Old Main Building, Groote Schuur Hospital, Main Road, Observatory 7925, South Africa. ${ }^{15}$ Department of Neurosurgery, Renji Hospital, Shanghai Institute of Head Trauma, Shanghai Jiaotong University School of Medicine, 1630 Dongfang Road, Shanghai 200127, China.

16 Johns Hopkins University School of Medicine, 1800 Orleans St. Sheikh

Zayed Tower, Baltimore, MD 21287, USA. ${ }^{17}$ Stanford Neuroscience Health Center, 213 Quarry Rd 4th FI MC 5958, Palo Alto, CA 94304, USA. ${ }^{18}$ Department of Neurosurgery, Pasteur Drive, Room R205, Edward's Building, MC 5327, Stanford, CA 94305, USA. ${ }^{19}$ Department of Neurological Surgery, School of Medicine, Case Western Reserve University, 11100 Euclid Avenue, HAN 5042 Cleveland, OH 44106, USA. ${ }^{20}$ Division of Neurosurgery, Department of Clinical Neurosciences, Addenbrooke's Hospital and University of Cambridge, Cambridge Biomedical Campus, Cambridge CB20QQ, UK. ${ }^{21}$ Department of Neurological Sciences, Christian Medical College, Ida Scudder Road, Vellore, Tamil Nadu, India. ${ }^{22}$ Vivian L Smith Department of Neurosurgery, McGovern Medical School at UTHealth, 6400 Fannin St, Suite 2800, Houston, TX 77030, USA. ${ }^{23}$ University of California San Francisco, San Francisco General Hospital and Trauma Center, 1001 Potrero Ave., Bldg 1, Room 101, San Francisco, CA 94110, USA. ${ }^{24}$ Neurology, K-11, Henry Ford Hospital, 2799 W Grand Blvd, Detroit, MI 48202, USA. ${ }^{25}$ Division of Anaesthesia, University of Cambridge and Addenbrooke's Hospital, Addenbrooke's Hospital, Hills Road, Box 93, Cambridge CB2 0QQ, UK. ${ }^{26}$ Department and Laboratory of Intensive Care Medicine, University Hospitals Leuven and KU Leuven, Herestraat 49, Box 7003 63, 3000 Leuven, Belgium. ${ }^{27}$ Oakland University William Beaumont School of Medicine, Beaumont Health, Michigan Head and Spine Institute, Southfield, MI, USA. ${ }^{28}$ Department of Intensive Care Medicine, CHUV-Lausanne University Hospital, University of Lausanne, Faculty of Biology and Medicine, Lausanne, Switzerland. ${ }^{29}$ Department of Neurosurgery, University of Pittsburgh Medical Center, Pittsburgh, PA, USA. ${ }^{30}$ Vanderbilt University Medical Center, Nashville, USA. ${ }^{31}$ Baylor College of Medicine, One Baylor Plaza, Houston, TX 77030, USA. ${ }^{32}$ Department of Neurosurgery, Alfred Hospital, Melbourne, Australia. ${ }^{33}$ Department of Surgery, Monash University, Melbourne, Australia. ${ }^{34}$ INUB/ MEDITECH Research Group, Neurosciences Institute, El Bosque University, Bogotá, Colombia. ${ }^{35}$ MEDITECH Foundation, Clinical Research, Calle 7-A \# 44-95, 760036 Cali, Colombia. ${ }^{36}$ University Hospital Vall D'Hebron, Barcelona, Spain. ${ }^{37}$ Department of Neurosurgery, Humanitas University and Research Hospital, Milan, Italy. ${ }^{38}$ World Federation of Neurosurgical Societies, Nyon, Switzerland. ${ }^{39}$ University of Pittsburgh Medical Center, 3550 Terrace St, Room 646, Pittsburgh, PA 15261, USA. ${ }^{40}$ Zuckerberg San Francisco General Hospital and Trauma Center, University of California, San Francisco, 1001 Potrero Ave., Ward 3A, San Francisco, CA 94110, USA. ${ }^{41}$ Department of Physiopathology and Transplantation, Milan University, Milan, Italy. ${ }^{42}$ Neuroscience Intensive Care Unit, Fondazione IRCCS Cà Granda Ospedale Maggiore Policlinico, Milan, Italy. ${ }^{43}$ Department of Intensive Care, Hospital Erasme, Université Libre de Bruxelles (ULB), Brussels, Belgium. ${ }^{44}$ Department of Neurological Surgery, Penn State University Milton S. Hershey Medical Center, 30 Hope Dr., Suite 1200 | Building B, Hershey, PA 17033, USA. ${ }^{45}$ Suruchi Bhargava Chair in Spinal 
Cord and Brain Regeneration Research, University of Ottawa, The Ottawa Hospital, C2 Neurosciences Unit, The Ottawa Hosptial, Civic Campus, 1053 Carling Avenue, Ottawa, ON K1Y 4E9, Canada. ${ }^{46}$ Department of Neurosurgery, Donald and Barbara Zucker School of Medicine At Hofstra/Northwell North, Shore University Hospital, 300 Community Drive, 9 Tower, Manhasset, NY, USA. ${ }^{47}$ Ronald Reagan UCLA Medical Center, UCLA Medical Center, Santa Monica, Santa Monica, USA. ${ }^{48}$ Posadas Hospital, Buenos Aires, Argentina. ${ }^{49}$ Emory University School of Medicine, 49 Jesse Hill Jr Dr, Atlanta, GA 30303, USA. ${ }^{50}$ School of Medicine and Dentistry, University of Rochester Medical Center, 601 Elmwood Ave, Box 655C, Rochester, NY 14642, USA. ${ }^{51}$ Section of Neurosurgery, University of Manitoba, GB1, 820 Sherbrook Street, Winnipeg, MB R3A 1R9, Canada.

\section{Acknowledgements}

We thank our moderators Kevin Dunne and Theodore Michalke, Jone Howard for our audience response system, Kelley Chaddock for extensive administrative support, and Peter Hendrickson, PhD, for managing our web-based surveys. We acknowledge the influence that the BOOST-3 Clinical Standardization Committee (Lori Shutter, Lisa Merck, Ramon Diaz-Arrastia, Rocco Armonda, Francis Bernard, Randall Chesnut, Anita Fetzick, Claude Hemphill, Luke James, Ryan Kitagawa, Carol Moore, David Okonkwo, Ava Puccio, Claudia Robertson, Uzma Samadani, Danielle Sandsmark, Robert Silbergleit) had on the generation of these protocols. We thank our financial supporters who include Adler/ Geirsch Attorney at Law, the American Association of Neurological Surgeons/ Congress of Neurological Surgeons Section on Neurotrauma and Critical Care, Bard, the Brain Trauma Foundation, DePuy, Hemedex, Integra, the Neurointensive Care Section of the European Society of Intensive Care Medicine, the Neurosurgery Society of Australasia, Medtronic, Moberg Research, Natus, Neuroptics, Raumedic, Sophysa, Stryker, and Zoll. PJH is supported by the NIHR (Research Professorship, Cambridge BRC and Global Health Research Group on Neurotrauma), and DKM is an Emeritus Senior Investigator of the National Institute of Health Research (UK).

\section{Compliance with ethical standards}

\section{Conflicts of interest}

On behalf of all authors, the corresponding author states that there are no relevant conflicts of interest.

\section{Open Access}

This article is licensed under a Creative Commons Attribution-NonCommercial 4.0 International License, which permits any non-commercial use, sharing, adaptation, distribution and reproduction in any medium or format, as long as you give appropriate credit to the original author(s) and the source, provide a link to the Creative Commons licence, and indicate if changes were made. The images or other third party material in this article are included in the article's Creative Commons licence, unless indicated otherwise in a credit line to the material. If material is not included in the article's Creative Commons licence and your intended use is not permitted by statutory regulation or exceeds the permitted use, you will need to obtain permission directly from the copyright holder. To view a copy of this licence, visit http://creativecommons.org/licen ses/by-nc/4.0/.

\section{Publisher's Note}

Springer Nature remains neutral with regard to jurisdictional claims in published maps and institutional affiliations.

Received: 16 November 2019 Accepted: 14 December 2019

Published online: 21 January 2020

\section{References:}

1. Hawryluk GWJ, Aguilera S, Buki A, Bulger E, Citerio G, Cooper DJ, Arrastia RD, Diringer M, Figaji A, Gao G, Geocadin R, Ghajar J, Harris O, Hoffer A, Hutchinson P, Joseph M, Kitagawa R, Manley G, Mayer S, Menon DK, Meyfroidt G, Michael DB, Oddo M, Okonkwo D, Patel M, Robertson C, Rosenfeld JV, Rubiano AM, Sahuquillo J, Servadei F, Shutter L, Stein D, Stocchetti N, Taccone FS, Timmons S, Tsai E, Ullman JS, Vespa P, Videtta W, Wright DW, Zammit C, Chesnut RM (2019) A management algorithm for patients with intracranial pressure monitoring: the Seattle International Severe Traumatic Brain Injury Consensus Conference (SIBICC). Intensive Care Med 45:1783-1794

2. Carney N, Totten AM, O'Reilly C, Ullman JS, Hawryluk GW, Bell MJ, Bratton SL, Chesnut R, Harris OA, Kissoon N, Rubiano AM, Shutter L, Tasker RC, Vavilala MS, Wilberger J, Wright DW, Ghajar J (2017) Guidelines for the management of severe traumatic brain injury, fourth edition. Neurosurgery $80: 6-15$

3. Okonkwo DO, Shutter LA, Moore C, Temkin NR, Puccio AM, Madden CJ, Andaluz N, Chesnut RM, Bullock MR, Grant GA, McGregor J, Weaver M, Jallo J, LeRoux PD, Moberg D, Barber J, Lazaridis C, Diaz-Arrastia RR (2017) Brain oxygen optimization in severe traumatic brain injury phase-ll: a phase II randomized trial. Crit Care Med 45:1907-1914

4. Kochanek PM, Tasker RC, Bell MJ, Adelson PD, Carney N, Vavilala MS, Selden NR, Bratton SL, Grant GA, Kissoon N, Reuter-Rice KE, Wainwright MS (2019) Management of pediatric severe traumatic brain injury: 2019 consensus and guidelines-based algorithm for first and second tier therapies. Pediatr Crit Care Med 20:269-279

5. Kochanek PM, Tasker RC, Carney N, Totten AM, Adelson PD, Selden NR, Davis-O'Reilly C, Hart EL, Bell MJ, Bratton SL, Grant GA, Kissoon N, ReuterRice KE, Vavilala MS, Wainwright MS (2019) Guidelines for the management of pediatric severe traumatic brain injury, third edition: update of the brain trauma foundation guidelines. Pediatr Crit Care Med 20:S1-S82

6. Hendrickson P, Pridgeon J, Temkin NR, Videtta W, Petroni G, Lujan S, Guadagnoli N, Urbina Z, Pahnke PB, Godoy D, Pinero G, Lora FS, Aguilera S, Rubiano AM, Morejon CS, Jibaja M, Lopez H, Romero R, Dikmen S, Chaddock K, Chesnut RM (2018) Development of a severe traumatic brain injury consensus-based treatment protocol conference in Latin America. World Neurosurg 110:e952-e957

7. Rosenthal G, Sanchez-Mejia RO, Phan N, Hemphill JC 3rd, Martin C, Manley GT (2011) Incorporating a parenchymal thermal diffusion cerebral blood flow probe in bedside assessment of cerebral autoregulation and vasoreactivity in patients with severe traumatic brain injury. J Neurosurg 114:62-70

8. Hebert PC, Wells G, Blajchman MA, Marshall J, Martin C, Pagliarello G, Tweeddale M, Schweitzer I, Yetisir E (1999) A multicenter, randomized, controlled clinical trial of transfusion requirements in critical care. Transfusion Requirements in Critical Care Investigators, Canadian Critical Care Trials Group. N Engl J Med 340:409-417

9. Robertson CS, Hannay HJ, Yamal JM, Gopinath S, Goodman JC, Tilley BC, Epo Severe TBITI, Baldwin A, Rivera Lara L, Saucedo-Crespo H, Ahmed O, Sadasivan S, Ponce L, Cruz-Navarro J, Shahin H, Aisiku IP, Doshi P, Valadka A, Neipert L, Waguspack JM, Rubin ML, Benoit JS, Swank P (2014) Effect of erythropoietin and transfusion threshold on neurological recovery after traumatic brain injury: a randomized clinical trial. JAMA 312:36-47

10. Morris GF, Juul N, Marshall SB, Benedict B, Marshall LF (1998) Neurological deterioration as a potential alternative endpoint in human clinical trials of experimental pharmacological agents for treatment of severe traumatic brain injuries. Executive Committee of the International Selfotel Trial. Neurosurgery 43:1369-1372

11. Chesnut RM, Temkin N, Carney N, Dikmen S, Rondina C, Videtta W, Petroni G, Lujan S, Pridgeon J, Barber J, Machamer J, Chaddock K, Celix JM, Cherner M, Hendrix T, Global Neurotrauma Research G (2012) A trial of intracranial-pressure monitoring in traumatic brain injury. N Engl J Med 367:2471-2481 